\title{
DER SCHIFFE HOCHSTIER DRÜMT. EINIGE BEMERKUNGEN ZU UNSEREM ÜBERSETZUNGSWORKSHOP
}

\author{
PAVEL NOVOTNÝ
}

Die poetische Übersetzung bietet einen ganz engen Kontakt mit dem Text: eine besonders intensive Art des Lesens. Soll die Übersetzung gelingen, so muss man dem Autor sozusagen auf die Finger gucken, seine Verfahrensweise und die formalen/inhaltlichen Prinzipien des Textes möglichst genau einsehen - und aufgrund dieser Einsicht den eigentlichen „Kern“ des Textes in die jeweilige Muttersprache kreativ übertragen. Dies heißt auch: der Ausgangspunkt einer guten Übersetzung ist eindeutig die Interpretation des Textes, keineswegs eine trockene, mechanische Übertragung des Inhalts. Versteht man den Text nicht, findet man dazu keinen guten Schlüssel, so hat man logischerweise auch keine Chance, eine gute Nachdichtung zu machen. Dies gilt sicher für die Literatur im Allgemeinen, am meisten jedoch eben für die Poesie.

Die deutsche Bezeichnung für ein poetisches Gebilde, nämlich das Gedicht, drückt diesen Anspruch auf Verständnis und Interpretation ja schon deutlich aus. Denn ein Gedicht soll vom Prinzip her einen möglichst konzentrierten, verdichteten Text darstellen, einen Text, in dem jedes Wort, ja häufig auch jede Silbe oder jeder Laut die gesamte Wirkung mitgestaltet. Nichts soll überflüssig bleiben, nichts soll fehlen, ein Gedicht ist im Idealfall so vollkommen wie ein Baum, eine Blume - oder, ja: zumindest wie ein gut funktionierendes Räderwerk. Auch die Übersetzung bzw. Nachdichtung sollte ein solches Ziel verfolgen, obwohl sie schließlich doch nur als ein formaler oder inhaltlicher Kompromiss erfolgen kann. Auf einer solchen Basis habe ich auch meinen Workshop konzipiert. Das Ziel war es, das jeweilige Gedicht möglichst genau zu analysieren und zu interpretieren, und dadurch die möglichen Übersetzungsmöglichkeiten zu entwerfen. Was auch als wichtig erschien, war das Phänomen der Unübersetzbarkeit bzw. die Einsicht, dass das Gedicht fest im dessen sprachlichen oder auch kulturellen Rahmen steckt, der sich häufig kaum in die Zielsprache übertragen lässt.

In unserem Workshop haben wir mit den Texten der Wiener Gruppe gearbeitet, speziell mit den Gedichten tom du tümmel von H. C. Artmann, weiter mit franz war Konrad Bayers und schließlich mit Artmanns poetischem Minidrama Die Fahrt zur Insel Nantucket. Da es in der Arbeitsgruppe sowohl tschechische als auch deutsche Studenten gab, habe ich die deutschen Teilnehmer innerhalb einzelner Gruppen als Sprachmittler benutzt, sodass die gemeinsame Übersetzungsarbeit als ein intensiver zweisprachiger Dialog verlaufen konnte.

Mit dem erstgenannten Gedicht habe ich mich schon früher mehrmals essayistisch beschäftigt (siehe die Quellenliste), denn ich halte diesen wenig bekannten Text für einen 
der besten Werke der neoavantgardistischen Dichtung überhaupt; tom du tümmel ist einerseits ein minimalistischer, konkretistisch angelegter Text, der jedoch die höchsten Prinzipien der Poesie bestätigt und sie zugleich ästhetisch umfunktioniert. Für den Übersetzer stellt dieses permutativ-rotierende Gedicht sicher eine große Herausforderung dar:

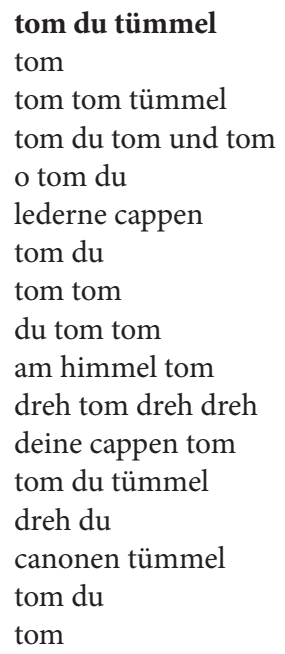

Mit den Studenten haben wir den Text Schritt für Schritt zerlegt und dessen phonetisch und rhythmisch bellenden, ja schießenden Rhythmus besprochen. Gemeinsam sind wir zu der Ansicht gekommen, der Text stelle eine Schlachtszene dar, die er zugleich formal selbst verkörpere: Kanonen und lederne Kappen kommen da vor, der Ausdruck tom (Name und Interjektion zugleich) gleicht durch seine rhythmische Anhäufung und ebenfalls durch die Aspiration des Explosiv-Lautes $(t)$ einer bellenden Tommy Gun - einem Maschinengewehr: der Mensch wird dadurch mit seiner Waffe gleichgesetzt, seine Funktion einer tötenden menschlichen Maschine wird durch den Namen direkt determiniert. Dies lässt sich ebenfalls im Zusammenhang mit dem klassischen Aufklärungsparadigma der Poesie verstehen (im Sinne „das Wort als Licht“), welches hier jedoch völlig auf den Kopf gestellt wird, indem das Gedicht - statt als Ausdruck des Lebens zu funktionieren - den allgegenwärtigen Tod (sowohl inner- als auch außerhalb des Textes) artikuliert.

Etwa in dieser Richtung erfolgte unsere gemeinsame Interpretation des Textes. Ferner haben wir uns auf die artikulatorische bzw. akustische Seite des Gedichtes konzentriert und den Anspruch erhebt, diese Ebene ebenfalls in der Übersetzung zu berücksichtigen. Nun ging es darum, den Text auf irgendeine Art und Weise ins Tschechische zu übertragen, und da sind etliche Probleme aufgetaucht, die die oben erwähnte These der Unübersetzbarkeit gut veranschaulichten - vor allem: der tschechische Vokativ tome (analog zu dem deutschen tom) würde den trommelnden, perkussiven Charakter des Textes auflösen, man müsse also entweder darauf verzichten oder nach einer anderen Lösung suchen. In meiner eigenen Übersetzung habe ich den Ausdruck tom durch rudo ersetzt, und so habe ich mich der Homonymie des Rudo und rudo (substantivische Form 
von rudý, also „das Feuer-“ oder „Blutrote“) bedient. So tritt in meiner Übersetzung ein gewisser Ruda (Rudolf) auf, der zugleich lautlich als blutrotes bzw. blut- und feuerbringendes Element wirkt. Ähnlich wie in dem Text von Artmann, in welchem Mensch und Waffe in einem Ausdruck vereint werden, wird hier der Name mit Blut, Feuer und der Destruktion gleichgesetzt:

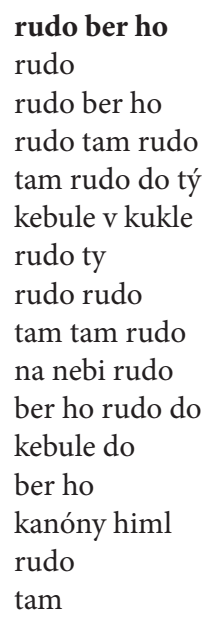

Diese meine Übersetzung habe ich den Studenten allerdings erst nach der Präsentation ihrer eigenen kreativen Leistungen vorgelegt, dabei freilich als keine Ideallösung, sondern nur als eine der vielen Möglichkeiten; was ich in meiner Übersetzung allerdings anstrebte, war ein durchkomponiertes, schlüssiges Gebilde, welches die Wirkung des Originaltextes zwar nicht restlos übertragen (dies wäre ja prinzipiell kaum möglich), dabei aber doch die Intentionen des Originaltextes beibehalten konnte.

Was die studentischen Übersetzungen angeht, so hat sich bestätigt, dass es auf der gemeinsamen interpretatorischen Basis unendlich viele Lösungen geben kann, dass es jedoch extrem schwierig ist, in kurzer Zeit eines Workshops eine gut zu funktionierende, eben schlüssige poetische Formel zu erfinden. Man könnte sogar sagen: je schlichter ein poetischer Text zu sein scheint, umso schwieriger lässt er sich in ein konzentriertes Gebilde transformieren. Hier zwei interessante Beispiele:

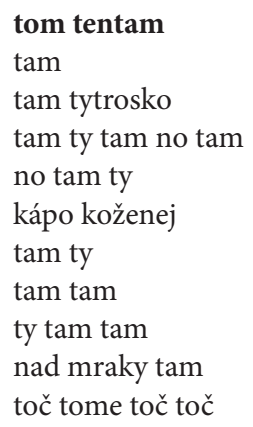




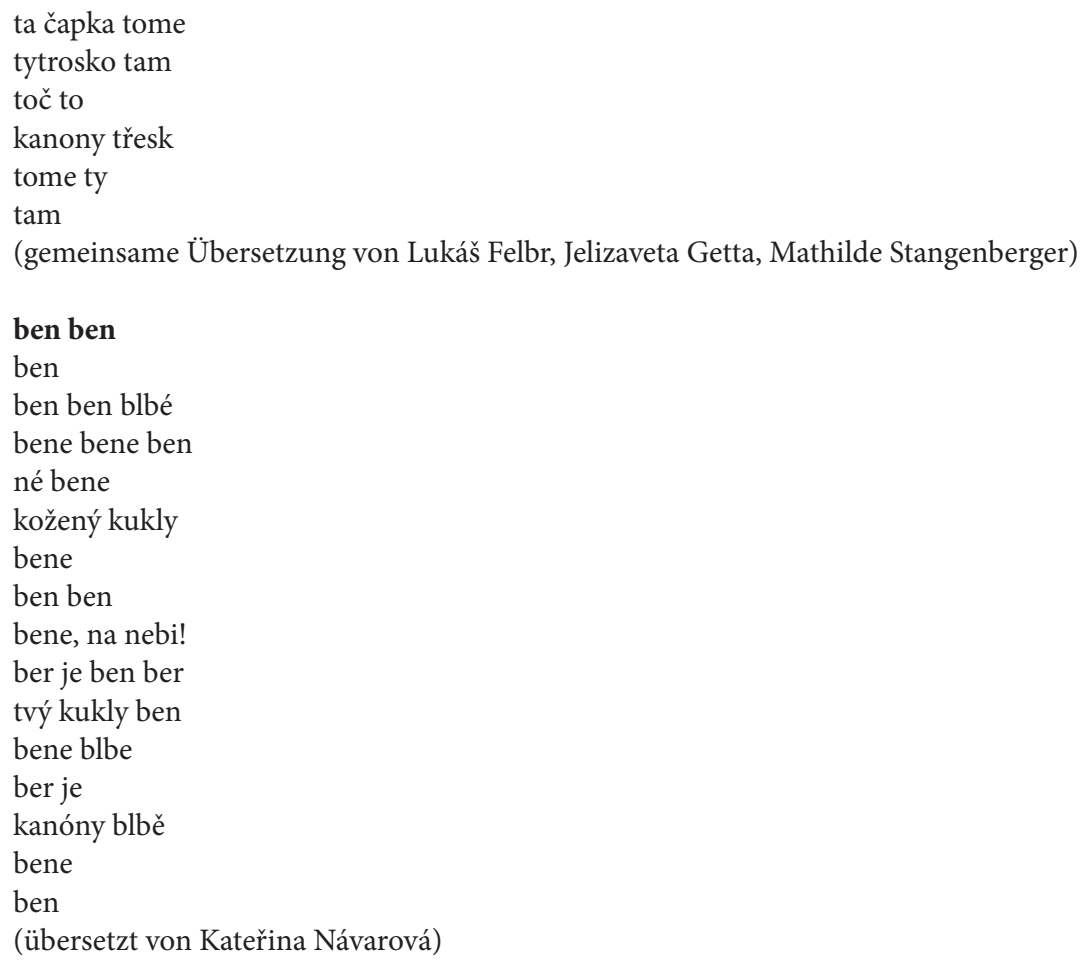

Während Artmanns Gedicht noch relativ viel Raum für die Übersetzung zulässt, so stellt Bayers Gedicht franz war einen Text dar, dessen sprachliche Schranken extrem eng sind, und der besonders aus formaler Sicht kaum mehr unübersetzbar ist:

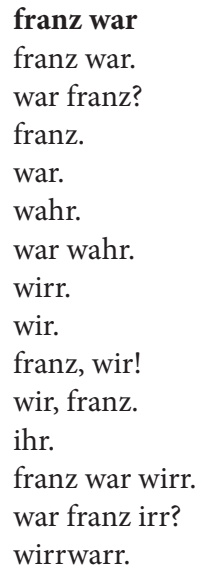

Auf den ersten Blick wirkt der Text ziemlich simpel: ein grammatischer und orthographischer Wirrwarr wird hier getrieben, eine freie Spielerei. Dieser Eindruck ändert 
sich aber grundsätzlich, stellt man sich die Frage, warum hier gerade ein gewisser Franz (also kein Hans, Richard oder etwa Erich) genannt wird. Denn eben in diesem Namen lässt sich der eigentliche Leitfaden erkennen, der dem ganzen grammatischen „Wirrwarr" einen tiefen und düsteren Sinn gibt. Bezieht man den Franz auf den berühmten Thronfolger Franz Ferdinand d'Este, so lässt sich das Gedicht als eine höchst konzentrierte zeit-räumliche Formel des Kriegschaos verstehen. Was die Übersetzung betrifft, so ist die härteste Nuß sicher der scheinbar einfache Titel bzw. der erste Vers des Gedichtes, wo sich eine wahrlich verhexte poetische Kontamination von Deutsch und Englisch erblicken lässt. Zwei Sprachen miteinander kontaminierend drückt der erste Vers total knapp die Ursache und zugleich ihre Konsequenz aus: denn die Folge davon, dass es den Franz nicht mehr gibt (also das er war und nicht ist), ist nichts anderes als the war. Aus diesem Ereignis erfolgt nun auch nur der ganze semantische „Wirrwarr“ des Textes oder auch der Welt, u. a. auch die Frage, die es seit jeher gab, ob der Franz denn irr oder auch wirr gewesen wäre (siehe dazu etwa den Thronfolger Ludwig Winders).

Zurecht haben die Studenten vor der extremen Textdichte dieses kurzen Textes kapituliert; und ich konnte ihnen ebenfalls kaum etwas anderes anbieten als eine lockere Variation, die im Grunde genommen nur wenig mit dem Originaltext zu tun hat und bloß auf den grammatischen Witz des Textes zielt; das Thema das Krieges kommt hier gar nicht vor, statt dessen wird hier mit der Grammatik und dem Namen Michal gerührt und gemischt:

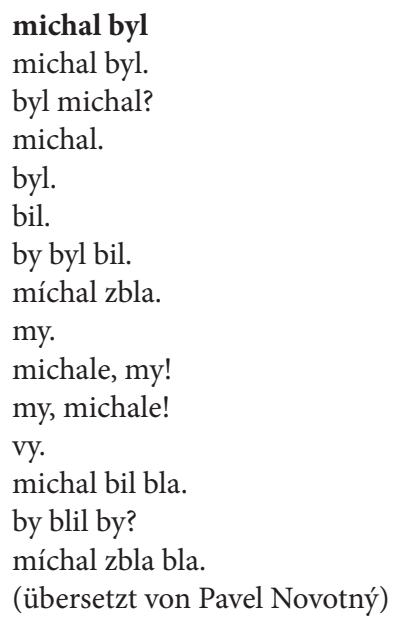

Im letzten Teil der Workshops haben wir uns mit der Fahrt zur Insel Nantucket von H. C. Artmann beschäftigt. Der Originaltext basiert auf einer durchaus abstrakten Vermischung des (Alt-)Deutschen mit dem Niederländischen und Englischen, wodurch eine poetisch-parodistische Seemannssprache entsteht. Teilweise kommen dazu noch orientalisch wirkende Sprachelemente (bagien und besam - siehe unten). Der Sinn des Ganzen ergibt sich aus den jeweiligen Wort-Fetzen und -Fragmenten sowie aus dem onomatopoischen, lautlichen Zusammenklang. Siehe den Ausschnitt: 


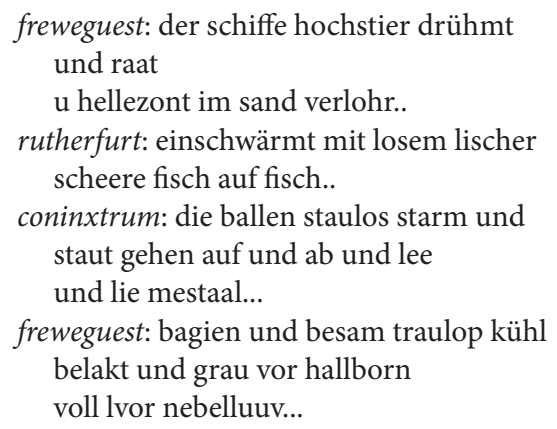

Gleich wie im Falle der oben genannten Gedichte ging es bei der Übersetzung darum, den spezifischen Sprachwitz dieses Textes in eine andere Sprache zu übertragen. Als die größte Schwierigkeit haben sich die sprachgeschichtlichen Faktoren erwiesen: denn das Deutsche, Niederländische und Englische passen ja schon bezüglich der Sprachfamilien bzw. der Sprachentwicklung gut zusammen (siehe u. a. die Ähnlichkeit des Plattdeutschen und des Niederländischen). Niederländisch und Tschechisch miteinander mischen zu wollen, wäre dagegen völlig unlogisch und sprachlich verdreht. Es würde sich also wohl anbieten, nur mit slawischen Sprachen zu arbeiten, die sich wohl problemlos zu einem panslawistischen poetischen Geplapper verbinden ließen - so ginge jedoch die spezifische abstrakte Seemänner-Rhetorik sicher verloren (...obwohl es auch unter den Slaven ja berühmte Seemänner gab, immerhin).

Aufgrund dieser Erwägungen sind wir zu dem Schluss gekommen, es wäre möglich das Tschechische bzw. das Slawische bloß mit dem Englischen zu vermischen; denn diese Sprachen sind zwar nicht direkt miteinander verwandt, beide sind jedoch im Stande, runde, wellenartige, schaukelnde Klänge zu produzieren und somit auch lautlich ineinanderzufließen: ein romantisierendes Tschechisch im Sinne von Mai K. H. Máchas käme ja dem diphthongischen, offen-vokalischen Englisch ganz gut entgegen. So entstünde ein gewisses metapoetisches „Czech-lisch“, das im Grunde genommen grenzenlose lautliche Spiele ermöglichen würde. Eben eine solche Mischung habe ich in meiner eigenen Übersetzung Artmanns Textes angestrebt, siehe den Ausschnitt:

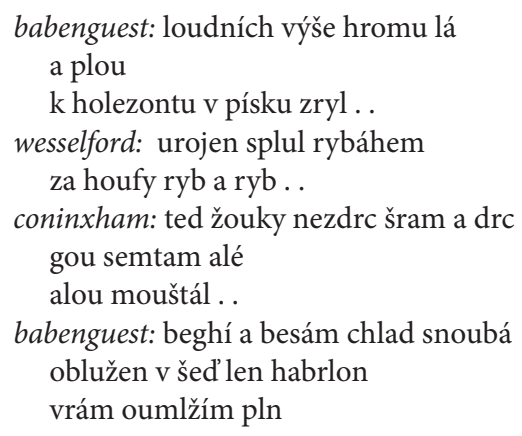


Hier ein Beispiel einer studentischen Übersetzung:

$\begin{array}{ll}\text { žeňgost: } & \begin{array}{l}\text { těch lodí sněžejň zasnívá } \\ \text { a pakrak }\end{array} \\ \text { feslbrod: } & \begin{array}{l}\text { soublačným pískem ztrácí se .. } \\ \text { tu ohejněna žralály } \\ \text { ryba rybu zdíhá . }\end{array} \\ \text { pankrol: } \quad \begin{array}{l}\text { a v závalu rejdí rybošum } \\ \text { šupí nahodoru }\end{array} \\ \quad \text { v návětrný bok } \\ \text { žeňgost: } \quad \begin{array}{l}\text { belga a daskar chladověrné } \\ \text { v šedokrytu tramburském } \\ \text { zamlhený zduch .. }\end{array} \\ \text { (Übersetzung als „Matoušová und Co.“ unterschrieben) }\end{array}$

Die Fahrt zur Insel Nantucket hat sich für die Übersetzung wiederum als besonders schwierig erwiesen. Denn bei aller Abstraktion des Originaltextes war es doch angebracht, dessen Wort-Fragmente, -Verzerrungen oder Kontaminationen gewissermaßen zu entziffern oder wenigstens die einzelnen semantischen Felder grob zu bestimmen; so hat man in jeder Gruppe rege diskutiert, was z. B. traulop oder besam denn heißen, oder wie ein nebelluv aussehen könnte. Schließlich ist man aber doch dazu gekommen, dass der eigentliche Sinn des Textes weniger im genauen Inhalt als im abstrakten und doch aussagekräftigen Klang des Ganzen besteht. Durch ein solches „Textverständnis“ kam man erst dazu, aufgrund des Textes frei zu dichten und komponieren, bzw. sein eigenes lautpoetisches Gebilde zu gestalten. Viele Studenten fühlten sich durch eine solche Freiheit paradoxerweise gehemmt, da sie an einen solcherlei breiten kreativen Raum kaum gewöhnt waren. So stellte dieser Workshop nicht nur einen translatologischen Exkurs, sondern auch eine kleine poetische Lektion dar.

Zum Abschied haben wir eine gemeinsame Audioaufnahme aller Übersetzungen von tom du tümmel gemacht, und zwar so, dass man den rotierenden Charakter des Textes in die akustische Form umsetzte: Im Flur der Leipziger Uni haben die Workshopteilnehmer einen Kreis gebildet und ihre Übersetzungen gemeinsam, übereinander laut und wiederholend rezitiert. In der Mitte des Kreises stand ich, in rotierender Bewegung das Stimmengewimmel stereophon mitschneidend. Eine gemeinsame, vielstimmige Montage ist dadurch entstanden, die sich meines Erachtens sehr gut anhört (sie wird auf meinen Webseiten ausgehängt und ist ebenfalls am Leipziger Lehrstuhl für Slawistik erreichbar).

\section{QUELLEN}

Rühm, Gerhard (Hg.): Die Wiener Gruppe. Achleitner, Artmann, Bayer, Rühm, Wiener. Erweiterte Neuausgabe, Reinbek: Rowohlt 1985, darin: tom du tümmel (S. 102), franz war (S. 139), die fahrt zur insel nantucket (S. 85-89).

Achleitner, Friedrich - Artmann, Hans Carl - Bayer, Konrad - Rühm, Gerhard - Wiener, Oswald: Wiener Gruppe. Praha: Rubato 2016 (übersetzt von Pavel Novotný und Nikola Mizerová), darin: rudo ber ho (S. 41), michal byl (S. 47), plavba k ostrovu nantucket (S. 48). 
Novotný, Pavel: Eine der vielen Übersetzungsmöglichkeiten. Zur Übersetzung des Gedichts tom du tümmel von H. C. Artmann. Dresden: Thelem Verlag 2016, S. 87-94.

Novotný, Pavel: Artmannova explozivní báseň (Artmanns explosives Gedicht), in: Souvislosti 4/2016, Prag, S. 4-9.

Dr. phil. habil. Pavel Novotný, Ph.D.

Katedra německého jazyka, Fakulta př́rodovědně-humanitní a pedagogická, Technická univerzita $v$ Liberci

pavel.novotny@tul.cz 